\title{
Uma Abordagem Participativa para Identificação de Preferências de Design de Crianças Autistas
}

\author{
Rallyson dos S. Ferreira ${ }^{1}$, Thaís H. Chaves de Castro ${ }^{1}$ \\ ${ }^{1}$ Instituto de Computação - Universidade Federal do Amazonas (UFAM) \\ Caixa Postal 15.064 - 91.501-970 - Manaus - AM - Brasil \\ rallysonferreira@ufam.edu.br, thaiseicomp.edu.br
}

\begin{abstract}
Neurotypical children, such as autistic children, do not participate in the software development process because they do not have sufficient language skills to express their design preferences. In this way, it is up to the designers to develop applications based only on the data obtained in the initial phase of the project. Through the participatory design process, it is possible to create approaches to improve understanding of the needs of this particular user group. Therefore, this research aims to develop an approach through a playful application aligned with the principles of inclusive design and participatory design for the recognition of a present user autistic project.
\end{abstract}

Resumo. Crianças neurotípicas, como é o caso de autistas, não participam do processo de desenvolvimento de softwares por não possuírem habilidades linguísticas suficientes para expressarem suas preferências de design. Dessa forma, cabe aos projetistas desenvolverem aplicativos com base, apenas, nos dados obtidos na fase inicial do projeto. Por meio do processo do design participativo é possivvel criar abordagens para melhorar a compreensão sobre as necessidades desse grupo de usuário em específico. Portanto, esta pesquisa objetiva o desenvolvimento de uma abordagem através de uma aplicação lúdica alinhada aos princípios de design inclusivo e design participativo para reconhecimento de preferências de design de usuários, inicialmente, autistas.

\section{Introdução}

Crianças neurotípicas, na tenra idade, como é o caso de crianças autistas (Transtorno do Espectro Autista - TEA), dependem diretamente de auxílio para realizar suas atividades do cotidiano. Sendo assim, essas dificuldades comprometem também as atividades de aprendizagem enfrentadas na escola [Castro et al. 2017].

Devido a esses problemas apresentados esse grupo de usuários ficam impossibilitados de participarem dos testes para coleta de dados no desenvolvimento de novas ferramentas computacionais criadas exclusivamente para este mesmo público. Isso ocorre devido à falta de capacidade cognitiva e habilidades linguísticas suficientes para participarem das atividades de forma ativa [APA 2014]. Dessa forma, torna-se difícil desenvolver aplicativos para auxiliar essas crianças, uma vez que não se podem obter informações sobre preferências de suas atividades e design adequado para utilizar nos softwares [Satterfield e Fabri 2017].

Ferramentas digitais podem promover a independência, o aprendizado, a qualidade de vida, e minimizar os problemas através dos processos de design inclusivo 
VIII Congresso Brasileiro de Informática na Educação (CBIE 2019)

Anais do XXX Simpósio Brasileiro de Informática na Educação (SBIE 2019)

(DI) e design participativo (DP). No entanto, a maioria dos aplicativos existentes são de propósitos gerais e não têm a flexibilidade exigida por grupos de usuários específicos [Britto e Pizzolato 2016].

Por intermédio da combinação dessas duas técnicas de design, DI e DP, é possível identificar as características sobre preferências de um determinado grupo de usuário em particular e propor o desenvolvimento de ferramentas com qualidade e projetadas para atender às suas necessidades [Lucke e Castro 2016].

Portanto, pela dificuldade de envolver usuários neurotípicos, como crianças com TEA nas idades iniciais, no processo de design (PD) este projeto de trabalho objetiva o desenvolvimento e avaliação de uma abordagem, alinhado com os preceitos de DI e DP para identificar preferências de usuário através de uma aplicação lúdica denominada "App TEA". Por meio dos resultados, esse projeto visa promover aos desenvolvedores informações relevantes a construção de ferramentas computacionais que possam se adequar as características e habilidades de um grupo específico de usuários.

\section{Trabalhos Relatados}

Esta seção aborda os principais trabalhos encontrados na literatura que estão alinhados com esta pesquisa e darão suporte para o andamento do projeto. Os assuntos relacionados envolvem aplicativos para crianças autistas, DI, DP e sistemas adaptativos.

Dessa forma, as principais propostas de trabalhos direcionadas ao público autista (crianças no caso) apresentam estudos com características inclusivas por meio do desenvolvimento de ambientes gráficos para fins de aprendizagem. Essas aplicações educacionais interativas têm por objetivo ajudar identificá-las na expressão facial e no sentimento que as envolve [Leite et al. 2019].

Em Castro e Lima (2017) o objetivo foi conceber formas inovadoras de projetar artefatos baseados em computador para crianças autistas. Esses artefatos foram inteligentes o suficiente para adaptar não apenas seus elementos de interface, mas também a maneira como eles interagem com usuários diferentes. Sendo assim é relatado um método estabelecido dentro de 3 abordagens chamadas de Princípios de Design para Interação Humano-Computador (IHC): Research Through Design (RtD), Participatory Design (PD) e Human Centred Design (HCD).

Através dos modelos de design participativo Satterfield e Fabri (2017) buscam formas de colocar o usuário como parte do processo de desenvolvimento de software e, assim, entender as suas reais necessidades, principalmente crianças com distúrbios cognitivos. Neste, é apresentado um estudo de caso com crianças autistas usando uma abordagem chamada "Modelo de Conectividade".

Utilizando técnicas de design inclusivo, o trabalho de Lucke e Castro (2016) enfatiza que o processo de desenvolvimento de sistemas inclusivos pode ajudar a identificar o potencial de reutilização e interoperabilidade dos sistemas desenvolvidos. Da mesma forma, Malinverni et al. (2016) propõem uma abordagem de DI para desenvolver jogos terapêticos. Para descrever este método, foi apresentado sua aplicação na concepção e desenvolvimento de um jogo baseado em Kinect para crianças com TEA de alto desempenho chamado "As Aventuras de Pico".

Modelos de design adaptativos foram propostos combinados com outras técnicas de design e modelos universais. Assim sendo, em Ribu e Patel (2016) o objetivo do projeto foi desenvolver soluções de aplicativos flexíveis e adaptáveis, orientadas para o usuário e que possam ajudar esse grupo diversificado a estruturar o seu dia a dia, por 
VIII Congresso Brasileiro de Informática na Educação (CBIE 2019)

Anais do XXX Simpósio Brasileiro de Informática na Educação (SBIE 2019)

exemplo, através de fotos de objetos e locais conhecidos pelo usuário. Embora a solução final seja projetada para um grupo de usuários específico, a flexibilidade integrada de sua estrutura e função significa que há o potencial inerente de abri-la para todos os tipos de usuários.

No trabalho de Braz, de Souza e Raposo (2014) foram adotadas 3 abordagens: Meta Design [Fischer 2013], Sondas de Design (Design Probes) [Mattelmaki 2016] e Engenharia Semiótica [De Souza 2005], que com a orientação de profissionais da área da saúde que trabalham com autismo, podem implementar tecnologias adaptáveis para auxiliar no atendimento desse público. Da mesma forma, o trabalho de Britto e Pizzolato (2016) descreve 107 potenciais de recomendações que foram normalizadas em 28 recomendações únicas e distribuídas em 10 categorias: (G01) Vocabulário Visual e Textual; (G02) Customização; (G03) Engajamento; (G04) Representações Redundantes; (G05) Multimídia; (G06) Resposta às Ações; (G07) Affordance; (G08) Navegabilidade; (G09) Estado do Sistema; (G10) Interação com Tela Sensível ao Toque.

Modelos de sistemas adaptativos também foram propostos para auxiliar o processo de ensino e aprendizagem [Silva e Dorça 2014]. Neste, são demonstrados resultados promissores obtidos através de experimentos com um método de adaptação que utiliza uma modelagem de preferências de aprendizagem representadas através de distribuições de probabilidades ao invés de tipos fixos. Seguindo este conceito, em Almeida, Breda e Santos (2016) é descrito um ambiente de aprendizagem desenvolvido sob este escopo universal, enfatizando os resultados obtidos durante as sessões de avaliação realizadas com usuários finais. O objetivo é avaliar um protótipo deste ambiente com características de adaptação dinâmica e projetado para apoiar $o$ desenvolvimento do raciocínio matemático em crianças autistas.

Diferente dos trabalhos relatados, este projeto de pesquisa coloca os usuários finais (crianças autistas) dentro do processo de desenvolvimento e realiza um estudo exploratório, através de uma aplicação mobile, com profissionais da educação e da saúde que lidam diretamente com as crianças, coletando preferências de design e adaptando os modelos por meio dos preceitos de DI e DP.

\section{Metodologia da Pesquisa}

Para um melhor entendimento o processo metodológico foi dividido em três etapas: (i) escolha do instrumento de diagnóstico base para o desenvolvimento do sistema; (ii) método de estudo diagnóstico para implementação e (iii) testes de inspeção.

\section{A. Avaliação Heurística}

Para identificação das falhas durante o processo de prototipação das telas do aplicativo App TEA foi realizado uma avaliação heurística [Nielsen 1994] das interfaces logo nas primeiras etapas. Dessa forma, foram convidados 3 avaliadores externos para aplicarem a avaliação heurística com o objetivo de evitar a participação dos próprios desenvolvedores.

Os resultados da avaliação heurística demonstram que o aplicativo possui interface simples, de fácil entendimento e funcionalidades agradáveis. No entanto, foram encontradas algumas discrepâncias que necessitaram de atenção para não causarem falhas durante a interação como: ícones sem função, botões sem utilidade, contraste de cores, visibilidade do estado do sistema, e algumas telas não tinham texto explicativo, que poderiam ser utilizados para um acompanhamento audiovisual. 
VIII Congresso Brasileiro de Informática na Educação (CBIE 2019)

Anais do XXX Simpósio Brasileiro de Informática na Educação (SBIE 2019)

Tabela 1. Resultado da Avaliação Heurística

\begin{tabular}{|c|c|c|}
\hline Heurística & Descrição & Severidade \\
\hline $\begin{array}{c}\text { 1. Severidade do } \\
\text { Estado do Sistema }\end{array}$ & O sistema não emite informação do estado atual & 2 - Leve \\
\hline $\begin{array}{c}\text { 4. Consistência de } \\
\text { Padrões }\end{array}$ & $\begin{array}{c}\text { Cores semelhantes foram utilizadas em ícones com } \\
\text { funções diferentes }\end{array}$ & $1-$ Cosmético \\
\hline $\begin{array}{c}\text { 10. Ajuda e } \\
\text { Documentação }\end{array}$ & $\begin{array}{c}\text { O sistema não oferece informações de quais passos } \\
\text { devem ser seguidos durante sua execução }\end{array}$ & 2 - Leve \\
\hline
\end{tabular}

A Tabela 1 demonstra, por meio da avaliação heurística, os erros detectados mais presentes. Dessa forma, os resultados apontaram erros de usabilidade de baixa prioridade, o que permitiu realizar as modificações, ainda durante a fase de desenvolvimento, evitando que esses erros fossem propagados a outras etapas. E além disso, aplicando as recomendações segundo [Lucke e Castro 2016], [Britto e Pizzolato 2016] e [Braz, de Souza e Raposo 2014] a aplicação sofreu alguns ajustes.

\section{B. Estudo Diagnóstico no Instituto Semear}

Para realizar os testes necessários com a aplicação, junto aos usuários e os especialistas, foram feitas visitas técnicas em uma instituição de ensino, o Instituto Semear em Manaus no estado do Amazonas, que atende crianças, jovens e adultos com Síndrome de Down, Autismo, Paralisia Celebral, entre outros.

O estudo diagnóstico iniciou com a aplicação de questionários a direção da instituição, profissionais que trabalham com as crianças e em seguida com os pais, com o objetivo de criar um perfil do instituto e de todos os envolvidos nesta pesquisa. $\mathrm{O}$ estudo contou com a participação de 11 profissionais ( 2 fonoaudiólogos, 2 psicólogos, 3 fisioterapeutas, 4 pedagogos) e 10 pais ou responsáveis pelos alunos. Todos os profissionais e pais possuem experiência no uso de tablets e smartphones, e tem familiaridade com recursos computacionais e aplicativos educacionais, o que facilitou a execução dos testes com o aplicativo. Antes de responderem o questionário os participantes leram e assinaram o TCLE (Termo de Consentimento Livre e Esclarecido).

Da mesma forma, através dos questionários, foram coletadas outras características do perfil das 10 crianças (público alvo) como idade, sexo, se possui dificuldade linguística para que fosse necessário o auxílio direto dos cuidadores ou profissionais para auxiliar nos testes, e experiência com smartphones ou tablets para saber se as crianças iriam conseguir utilizar o dispositivo adequadamente. Portanto, a Tabela 2 demonstra o perfil de cada criança participante do teste.

Tabela 2. Perfil das Crianças Autistas

\begin{tabular}{|c|c|c|c|c|c|}
\hline Aluno & Idade & $\begin{array}{c}\text { Grau de } \\
\text { Severidade }\end{array}$ & Sexo & $\begin{array}{c}\text { Dificuldade } \\
\text { Linguística }\end{array}$ & $\begin{array}{c}\text { Experiência com } \\
\text { Celular/Tablets }\end{array}$ \\
\hline A1 & 4 & Médio & $\mathrm{M}$ & Não & Não \\
\hline A2 & 4 & Médio & M & Não & Sim \\
\hline A3 & 4 & Médio & M & Não & Não \\
\hline A4 & 5 & Alto & M & Sim & Não \\
\hline A5 & 5 & Médio & F & Não & Sim \\
\hline A6 & 5 & Médio & M & Não & Sim \\
\hline A7 & 5 & Médio & M & Não & Sim \\
\hline A8 & 6 & Alto & F & Não & Não \\
\hline A9 & 6 & Médio & M & Não & Sim \\
\hline A10 & 7 & Médio & M & Não & Sim \\
\hline
\end{tabular}


VIII Congresso Brasileiro de Informática na Educação (CBIE 2019)

Anais do XXX Simpósio Brasileiro de Informática na Educação (SBIE 2019)

De acordo com a Tabela 2 a maioria dos alunos possuem grau de severidade médio de autismo, o que foi importante para facilitar a realização dos testes uma vez que aumenta as chances de observar as características desses usuários. Outra característica observada (Tabela 2) é sobre a experiência no uso de celular/tablets em que, apenas 4 alunos não utilizam com frequência os aparelhos. No entanto, os pais declararam que as crianças já tiveram contato e sabem manusear os dispositivos.

Através dos questionários, foi constatado também, através da experiência dos profissionais e familiares das crianças autistas, algumas características sobre preferência das crianças quanto ao uso de softwares de aprendizagem e comunicação voltados para autistas. Foram criados três questionários específicos para a direção da escola, profissionais e pais e continham questões como:

- Você acredita que seu(sua) filho(a) lembra melhor das coisas vendo imagens/representações gráficas, lendo texto ou ouvindo áudio?

- Imagine se seu filho pudesse informar suas preferências de aplicativos como: as cores preferidas, sons, tipos de jogos, dispositivo utilizado (celular, tablets, notebooks ou PC), etc. Com sua experiência, quais características você acredita que ele(a) indicaria?

Sendo assim, após análise dos questionários foi possível desenvolver a primeira versão do aplicativo App TEA e realizar os primeiros testes de usabilidade.

\section{Testes de Inspeção com a Aplicação}

Para identificação das falhas de interação e validação do App TEA, foi realizado uma avaliação de inspeção do protótipo com o objetivo de verificar se o aplicativo desenvolvido está em um nível aceitável de usabilidade do ponto de vista dos especialistas em interação. Os testes de inspeção de software é uma técnica de análise estática para verificar propriedades de qualidade de produto de software, é bem estruturada e possui processos e regras bem definidos, técnicas de leitura para detecção de defeitos e resultados documentados [Sommerville 2011].

Na pesquisa foi utilizada a Técnica de Leitura Baseada em Perspectivas pelo fato de ser mais específica e direcionada, o que torna a inspeção mais simples de ser aplicada [Briand, Freimut e Vollei 2000]. Portanto, após o detalhamento do projeto a toda equipe de inspeção cada integrante avaliou o aplicativo de acordo com a sua experiência na área que atua e do ponto de vista dos usuários (crianças autistas).

Tabela 3. Lista de Defeitos Coletados Durante o Teste de Inspeção

\begin{tabular}{|c|c|c|c|}
\hline Classe & Subclasse & Registro & Descrição \\
\hline \multirow{4}{*}{ 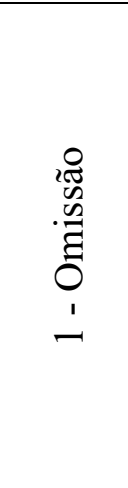 } & $\begin{array}{l}\text { 1. Funcionalidade } \\
\text { Omitida (FO) }\end{array}$ & $\begin{array}{l}\text { A imagem de localização da } \\
\text { tela não está ativa. }\end{array}$ & $\begin{array}{l}\text { A figura de localização do aplicativo, parte } \\
\text { superior esquerdo, não tem funcionalidade. } \\
\text { Os inspetores tentaram utilizar como atalho } \\
\text { para passar para outra cena. }\end{array}$ \\
\hline & $\begin{array}{l}\text { 2. Performance } \\
\text { Omitida }(\mathrm{PO})\end{array}$ & Posição da tela. & $\begin{array}{l}\text { O usuário visualiza melhor a aplicação } \\
\text { quando o smartphone está na horizontal. } \\
\text { Sugere-se modificar para modo paisagem. }\end{array}$ \\
\hline & $\begin{array}{l}\text { 3. Ambiente } \\
\text { Omitido (AO) }\end{array}$ & $\begin{array}{l}\text { Informações sobre a equipe } \\
\text { de desenvolvimento não } \\
\text { aparece no documento. }\end{array}$ & $\begin{array}{l}\text { A tela de ajuda tem uma breve descrição do } \\
\text { documento, mas, não tem informações sobre } \\
\text { a equipe de desenvolvimento. }\end{array}$ \\
\hline & $\begin{array}{l}4 . \\
\text { Omitida (IO) }\end{array}$ & & (2. \\
\hline
\end{tabular}


VIII Congresso Brasileiro de Informática na Educação (CBIE 2019)

Anais do XXX Simpósio Brasileiro de Informática na Educação (SBIE 2019)

\begin{tabular}{|c|c|c|c|}
\hline \multirow{4}{*}{ 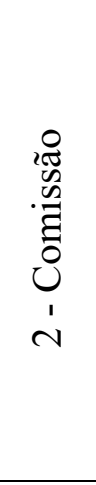 } & $\begin{array}{l}\text { 1. Informação } \\
\text { Ambígua (IA) }\end{array}$ & $\begin{array}{l}\text { O ícone de ativar e } \\
\text { desativar o som muda o tipo } \\
\text { somente nas configurações. }\end{array}$ & $\begin{array}{l}\text { Se torna confuso onde encontrar o tipo de } \\
\text { som para que seja alterado. É viável } \\
\text { informar onde está essa funcionalidade. }\end{array}$ \\
\hline & $\begin{array}{l}2 . \quad \text { Informação } \\
\text { Inconsistente (II) }\end{array} \mid$ & $\begin{array}{l}\text { As imagens dos personas } \\
\text { devem ser caracterizadas } \\
\text { como desenhos de crianças. }\end{array}$ & $\begin{array}{l}\mathrm{Na} \text { tela de escolher personas as imagens } \\
\text { possuem figuras de pessoas adultas, quando } \\
\text { deveriam possuir personagens para crianças. }\end{array}$ \\
\hline & $\begin{array}{l}\text { 3. Funcionalidade } \\
\text { Incorreta (FI) }\end{array}$ & $\begin{array}{l}\text { Customização não está } \\
\text { ativo. }\end{array}$ & $\begin{array}{l}\text { As funcionalidades de cores do aplicativo e } \\
\text { tamanho da fonte não estão funcionando, } \\
\text { como deveriam. }\end{array}$ \\
\hline & $\begin{array}{l}\text { 4. Seção Errada } \\
(\mathrm{SE})\end{array}$ & $\begin{array}{l}\text { O vídeo deve estar na tela } \\
\text { inicial. }\end{array}$ & $\begin{array}{l}\text { O vídeo sobre o "como utilizar o aplicativo" } \\
\text { está localizado na tela de ajuda, tornando-se } \\
\text { imperceptível ao usuário. }\end{array}$ \\
\hline$m \frac{2}{0}$ & $\begin{array}{l}\text { Defeitos que não } \\
\text { se enquadram nos } \\
\text { tipos acima. }\end{array}$ & $\begin{array}{l}\text { A sala de aula não possuía } \\
\text { personas para } \\
\text { inseridos. }\end{array}$ & $\begin{array}{l}\text { As crianças tem opção de escolher personas, } \\
\text { mas na sala de aula não tem essa opção. É } \\
\text { interessante para as crianças interagir com as } \\
\text { personas que elas se identificam. }\end{array}$ \\
\hline
\end{tabular}

O processo de inspeção formal inclui seis etapas principais [Fagan 1986], [Wong 2002], cada qual com objetivos específicos: Planejamento, Visão Geral, Preparação, Realização da Inspeção, Retrabalho e Revisão.

A Tabela 3 exemplifica as observações descritas pelos inspetores de forma detalhada, visando uma inspeção qualitativa do design. Assim, não demonstramos os valores quantitativos dos defeitos encontrados, somente o relato da análise para avaliação dos erros que foram considerados mais graves.

\section{Estudo Exploratório}

O estudo exploratório é um teste para avaliar a percepção do usuário sobre um projeto [Bach 2004]. Neste trabalho, o estudo exploratório foi utilizado para avaliar a qualidade de interação dos usuários com a ferramenta App TEA. Dessa forma, através do protótipo inicial foi possível obter características importantes sobre a experiência de uso, interação e receptividade com o aplicativo. Com isso, também foram coletadas informações quanto à utilidade e facilidade de uso da ferramenta.

O novo design obtido através dos métodos de avaliação heurística, estudo diagnóstico e os testes de inspeção com a aplicação geraram um novo modelo a ser aplicado com as crianças no estudo exploratório.

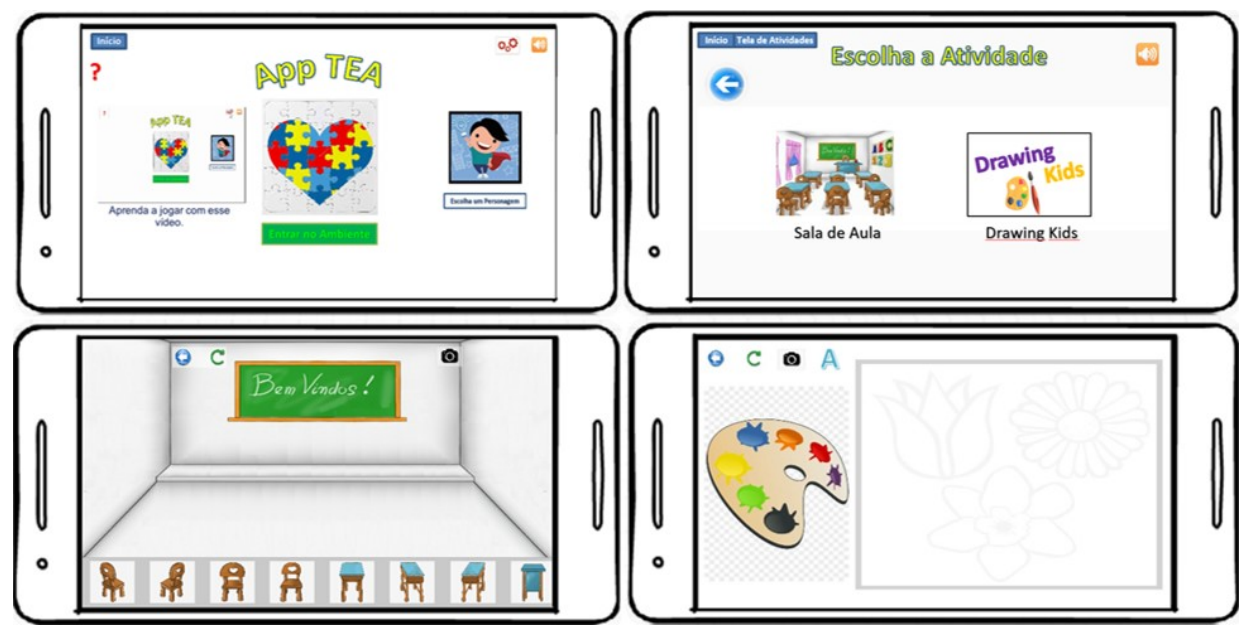

Figura 1. Tela Inicial, Tela de Escolha de Atividade, Tela de Atividade Sala de Aula, Tela de Atividade Drawing Kids. 
VIII Congresso Brasileiro de Informática na Educação (CBIE 2019)

Anais do XXX Simpósio Brasileiro de Informática na Educação (SBIE 2019)

Dessa forma, detalhamos os passos a serem executados no aplicativo como visto na Tabela 4 a seguir.

Tabela 4. Execuções a Serem Realizadas no Aplicativo

\begin{tabular}{|l|l|}
\hline 01 & Executar o vídeo de demonstração do App TEA \\
\hline 02 & Escolher uma persona ou escolher tirar uma foto \\
\hline 03 & Entrar na Atividade Sala de Aula \\
\hline 04 & Inserir objetos na sala de aula \\
\hline 05 & Escolher mudar ou desativar o som \\
\hline
\end{tabular}

As crianças tiveram o acompanhamento dos pais e dos profissionais para que pudessem orientar o uso do aplicativo e dessa forma proceder de forma correta com o seu uso, como também, observar o comportamento das crianças durante os testes.

Durante os testes, foram dispostos 2 avaliadores (desenvolvedores), 2 profissionais (professor do aluno e 1 fisioterapeuta) e o cuidador (Pai responsável pela criança). No total realizaram os testes 10 crianças, que segundo Nielsen (1994) é um número aceitável de amostras.

Durante o estudo exploratório, identificamos alguns benefícios que o uso do App TEA pode trazer a pesquisa. Entre os benefícios, está à facilidade de trabalhar atividades comuns em que as crianças com autismo estão diariamente exercendo. Os profissionais do Instituto Semear juntamente com os pais observaram os alunos utilizando o aplicativo e assim expressaram seu ponto de vista em relação à usabilidade da ferramenta. Isso foi importante para que realmente houvesse a validação da aplicação. Por isso, ao final do teste foi realizada uma entrevista com todos os envolvidos para avaliar a qualidade de uso do App TEA.

Através do estudo foi possível observar que os usuários tiveram facilidade para aprender a utilizar e ganhar habilidades de uso durante a execução. Nas palavras dos profissionais "A estrutura da interface é simples e autoexplicativa, pela consistência com padrões já conhecidos, o reconhecimento das funções de cada botão foi uma tarefa fácil". Outro profissional se mostrou animado em relação ao app conseguir prender a atenção das crianças "ele consegue chamar e prender a atenção das crianças com autismo e assim irá ajudar em seu objetivo".

Em relação à atividade "Sala de Aula" o objetivo é identificar preferências quanto a organização de espaço, objetos e convivência. Os relatos informados pelos entrevistados demonstram que a atividade irá auxiliar a representar o espaço físico em que diariamente as crianças participam, e dessa forma, poderão compreender e organizar de acordo com a sugestão dos alunos.

A atividade "Drawing Kids" faz representação a técnica chamada Brain Draw [Melo, Baranauskas e Soares 2008]. O Brain Drawn visa obter representações das crianças por meio de desenhos em que as mesmas sublinham os traços par formar as imagens. O Drawing Kids, além de ter uma prancha de desenhos a disposição para serem inseridos e sublinhados, possui também a opção de forma livre, outra prancha de preencher cores, inserir texto, e a exemplo da atividade Sala de Aula, tirar uma foto da imagem formada.

De maneira geral, houve uma boa aceitação em relação às atividades encontradas no app. Segundo os pais "é interessante poder acompanhar e perceber algumas formas de organização das crianças. Isso é ótimo para entender o que eles 
VIII Congresso Brasileiro de Informática na Educação (CBIE 2019)

Anais do XXX Simpósio Brasileiro de Informática na Educação (SBIE 2019)

imaginam na forma física esses espaços e formas". Esse feedback é importante para que além de repensar a forma de aperfeiçoar as atividades, também se possa criar novas atividades. Durante a entrevista, a pedagoga do instituto relatou "sabendo dos objetivos do aplicativo para coletar preferências de usuário utilizando crianças com autismo, as atividades podem nos ajudar no dia a dia através de exemplos com espaços e formas fisicas".

Ao final da entrevista os pais e profissionais questionaram a utilização do App TEA por crianças com outros tipos de transtornos cognitivos, usando modelos baseados em Design Universal, e assim ser usado por todos.

\section{Resultados e Trabalhos Futuros}

Devido às dificuldades de comunicação e interação social, crianças com TEA não participam do desenvolvimento de aplicativos, o que dificulta a coleta de dados uma vez que não se consegue entender qual o design ideal e as preferências de usuário para se construir softwares adequados para esse público. Sendo assim, este trabalho apresentou um estudo referente ao desenvolvimento de uma aplicação mobile voltada, inicialmente, para o público autista para identificação de preferências de usuários utilizando os conceitos de DI e DP, o App TEA.

Os resultados mostram que ao utilizar técnicas baseadas no Processo de Design Inclusivo [Lucke e Castro 2016];[Marins 2011], Design Participativo [Melo, Baranauskas e Soares 2008], como também Design Universal [Hayashi 2014] que permitam aos alunos, com autismo, demonstrar questões sobre preferências, os desenvolvedores de software conseguirão coletar dados qualitativos úteis para auxiliar no desenvolvimento de seus projetos, voltados principalmente para públicos, antes considerados incapazes de participar do processo de software. Portanto, apesar de se tratar de uma aplicação que foi desenvolvida e testada em um espaço de tempo, consideravelmente curto, os resultados demonstram êxito em seu emprego, além de apresentar-se como um aplicativo simples, de fácil entendimento e percepção.

A pesquisa também contribui para o desenvolvimento de ferramentas computacionais mais autônomas e inteligentes, com características de adaptabilidade, podendo se adequar as habilidades ou necessidades de um determinado grupo de usuário, enriquecendo as ações de acessibilidade e de pesquisas dentro dessa área.

Para trabalhos futuros pretende-se idealizar outras atividades para coleta de dados sobre preferência de usuários autistas e, dessa forma, tornando o aplicativo mais amplo e com outras características funcionais, como também, mais interativo possibilitando que os usuários interajam entre si. Além disso, disponibilizar testes com grupos de crianças com outros transtornos cognitivos e assim, torná-lo de propósito geral. E quanto as atividades apresentadas no App TEA, como no caso da atividade Sala de Aula, deseja-se realizar uma validação dessas atividades na prática, para constatar se realmente as crianças reproduzem fisicamente a organização dos espaços virtualmente e qual o comportamento das mesmas ao alterar esses espaços.

\section{Agradecimentos}

Especialmente a Instituição de Ensino SEMEAR pelo grande apoio e receptividade, na pessoa da diretora e profissionais que atuam com seriedade e amor para com as 
VIII Congresso Brasileiro de Informática na Educação (CBIE 2019)

Anais do XXX Simpósio Brasileiro de Informática na Educação (SBIE 2019)

crianças. Assim também, aos pais pela colaboração, tempo para nos atender e principalmente por confiar na nossa equipe para realizar os testes com suas crianças.

Agradecemos a FAPEAM (Fundação de Amparo a Pesquisa do Estado do Amazonas) pelo apoio financiamento a pesquisa.

\section{Referências}

Almeida A., Breda A., and Santos M. (2016) "Learning Environment for autism spectrum disorder: a universal approach to the promotion of mathematical reasoning". In: Procedings of the 7th International Conference on Software Development and technologies for Enhancing Accessibillity and fighting. InfoExclusión 162-169.

American Psychiatric Association. (2014). "DSM-5: manual diagnóstico e estatístico de transtornos mentais". 5. ed. Porto Alegre: Artmed.

Bach, J. (2004). "Exploratory Testing, in The Testing Practitioner". Second ed., E. van Veenendaal Ed., Den Bosch: UTN Publishers, pp. 253-265.

Braz, Priscila, de Souza, Clarisse S., Raposo, Alberto. (2014). "Design de Tecnologias Adaptáveis para Uso de Profissionais da Área de Autismo". Brazilian Symposium on Human Factors in Computing Systems. October 27-31, 2014, Foz do Iguaçu, PR, Brazil.

Briand, L.C.; Freimut, B.; Vollei, F. (2000). "Assessing the Cost-Effectiveness of Inspections by Combining Project Data and Expert Opinion". IEEE Transactions on Software Engineering, n. 10, vol. 22, p. 124, 2000.

Britto, Talita C. P., Pizzolato, Ednaldo Brigante. (2016). "Gaia: uma proposta de um guia de recomendações de acessibilidade de interfaces web com foco em aspectos do autismo". V Cngresso Brasileiro de Informática na Educação (CBIE 2016).

Castro, Thaís, Castro, Alberto, Lima, Lima, David, and Bjorn, P. (2017). "Model Playground for Autistic Children: Teaching Social Skills through Tangible Collaboration". IEEE 17th International Conference on Advanced Learning Technologies (ICALT), Timisoara, 2017, pp. 441-445.

Castro, Thaís, Lima, David. (2017). Designing for Children Using the RtD and HCD Approaches". In: Antona M., Stephanidis C. (eds) Universal Access in HumanComputer Interaction. Design and Development Approaches and Methods. UAHCI 2017.

De Souza, Clarisse S. (2005). "The semiotic engineering of human-computer interaction". Cambridge, Mass.: The MIT Press.

Fagan, M. E. Advances in Software Inspections. (1986). "IEEE Transactions on Software Engineering". n. 7, vol. 12, p. 744-751, 1986.

Fischer, Gerhard. (2013). "Meta-Design: Empowering all stakeholder as codesigners". In: R. Luckin, P. Goodyear, B. Grabowski, S. Puntambeker, J. Underwood, \& N. Winters (Eds.), Handbook on Design in Educational Computing, Routledge, London, pp. 135-145.

Hayashi, Elaine C. S., Martins, Ewerton M., Duarte, Robson E., Martins, Claudinei, Costa, Henrique M., e Duarte, Sergio H. Mourthé. (2014). "Design universal 
VIII Congresso Brasileiro de Informática na Educação (CBIE 2019)

Anais do XXX Simpósio Brasileiro de Informática na Educação (SBIE 2019)

em Touchscreens: um modelo de interação acessível”. In Companion Proceedings of the 13th Brazilian Symposium on Human Factors in Computing Systems (IHC '14). Sociedade Brasileira de Computação, Porto Alegre, Brazil, Brazil, 53-54.

Leite J., Maia I., Ferreira A., Rosa L. (2019) “Graphic Design of Interactive Tools for People with Autistic Spectrum Disorders". In: Di Bucchianico G. (eds) Advances in Design for Inclusion. AHFE 2018. Advances in Intelligent Systems and Computing, vol 776. Springer, Cham.

Lucke, Ulrike, Castro, Thais. (2016). “The Process of Inclusive Design”. In IEEE 16th International Conference on Advanced Learning Technologies. pp. 446-447.

Malinverni L., Mora-Guiard J., Padillo V., Valero L., Hervás A., Pares N., (2016) "An inclusive design approach for developing video games for children with autism spectrum disorder", Computers in Human Behavior.

Marins, Simone C. F. (2011). "Design Universal, Acessibilidade e Tecnologia Assistiva: a formação profissional do terapeuta ocupacional na perspectiva da equidade". Tese (Doutorado). Programa de Pós-Graduação em Educação Especial. Universidade Federal de São Carlos, São Carlos, 2011, 246 p.

Mattelmäki, Tuuli. (2006). "Design Probes". University of Art and Design Helsinki, Helsinki.

Melo, A. M.; Baranauskas, M. C. C.; Soares, S. C. de M. (2008) "Design com Crianças: da Prática a um Modelo de Processo", Revista Brasileira de Informática na Educação, v. 16, n. 1, 43-55.

Nielsen, J. (1994). “Avaliação heurística”. Em Nielsen, J. e Mack, RL (Eds.), Usability Inspection Methods . John Wiley \& Sons, Nova York, NY.

Ribu, K., Patel, T. (2016) "Developing a user-centred planning tool for young adults with development disorders: A research-based teaching Project". In: Studies in Health Technology and Informatics.

Satterfield, Debra e Fabri, Marc. (2017). "User Participatory Methods for Inclusive Design and Research in Autism: A Case Study in Teaching UX Design”. 186-197. 10.1007/978-3-319-58634-2_15.

Silva, Daydson and Dorça, Fabiano. (2014). "Uma Abordagem Automática para Personalização do Processo de Ensino Baseada em Estilos de Aprendizagem em Sistemas Adaptativos e Inteligentes para Educação a Distância". Revista Brasileira de Informática na Educação, Volume 22, Número 2, 2014.

Sommerville, I. (2011). "Engenharia de Software”. 9a Edição. Pearson Education, 2011.

Wong, Y. K. (2002). "Use of Software Inspection Inputs in Pratice". Communications of the ACM, n. 8, vol. 35, p. 725-726, 2002. 